\title{
FRATURAS DO CÔNDILO OCCIPITAL: ATUALIZAÇÃO DA EXPERIÊNCIA EM NOSSO SERVIÇO E REVISÃO DA LITERATURA
}

\author{
FRACTURES OF THE OCCIPITAL CONDYLE: UPDATED EXPERIENCE IN OUR SERVICE \\ AND LITERATURE REVIEW
}

\author{
FRACTURAS DEL CÓNDILO OCCIPITAL: ACTUALIZACIÓN DE LA EXPERIENCIA \\ EN NUESTRO SERVICIO Y REVISIÓN DE LA LITERATURA
}

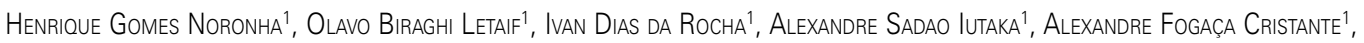
Reginaldo Perilo de Oliveira', Tarcísio Eloy Pessoa de Barros Filho'

\begin{abstract}
RESUMO
Apresentamos neste trabalho a casuística de fraturas de côndilo occipital diagnosticada e tratada em nosso serviço nos últimos 10 anos. 0 presente estudo dá continuidade ao levantamento epidemiológico já realizado no período de 1993 a 2000 . No período de 2001 a 2011 , foram diagnosticados seis casos de fraturas de côndilo occipital e todos eles foram tratados de forma conservadora, exceto um, no qual se colocou um halo craniano inicialmente. Nos cinco casos tratados de modo conservador, observaram-se bons resultados, com estabilidade do segmento nas radiografias funcionais, ausência de limitação de movimento e ausência de dor. O paciente que foi tratado com halo apresentava tetraplegia parcial FRANKEL C e fraturas associadas (C5, C6, L1 e L2) e não apresentou melhora do quadro neurológico, verificando-se limitação do movimento cervical. Destacamos ainda a importância da investigação ativa de lesões na transição occipitocervical, principalmente nos casos decorrentes de trauma de alta energia, pois além das implicações clínicas da detecção precoce da fratura, o melhor emprego dos exames subsidiários tem sido um fator relevante para o aumento da incidência de fraturas do côndilo occipital. Como é historicamente relatado, o tratamento conservador ainda é eficaz e com baixo índice de complicações, e o tratamento cirúrgico deve ser indicado em casos com instabilidade definida.
\end{abstract}

Descritores: Osso occipital; Traumatismos craniocerebrais; Humanos.

\begin{abstract}
We present a series of occipital condyle fractures diagnosed and treated in our hospital during the past 10 years. The present study continues an epidemiologic survey already performed in the period 1993-2000. From 2001 to 2011, six cases of fracture of the occipital condyle were diagnosed and all of them were treated conservatively, except one, in which a halo was initially placed. In the five cases treated conservatively, there were good results, with stable segment in functional radiographs, absence of limitation of motion and no pain. The patient treated with halo had FRANKEL C partial quadriplegia and associated fractures (C5, C6, L1, and L2), showing no neurological improvement, besides observing cervical motion limitation. We also highlight the importance of active research on lesions in the occipital-cervical transition, particularly in cases resulting from high-energy trauma, because in addition to the clinical implications of early detection of fracture, the best use of ancillary tests have been an important factor for the increased incidence of fractures of the occipital condyle. As is historically reported, conservative treatment is still effective and has a low rate of complications, and surgical treatment should be indicated in cases with established instability.
\end{abstract}

Keywords: Occipital bone; Craniocerebral trauma; Humans.

RESUMEN

Presentamos en este trabajo la casuística de fracturas de cóndilo occipital diagnosticada y tratada en nuestro servicio en los últimos 10 años. El presente estudio le da continuidad al levantamiento epidemiológico ya realizado en el período de 1993 a 2000. En el período de 2001 a 2011, fueron diagnosticados seis casos de fracturas de cóndilo occipital y todos ellos fueron tratados de forma conservadora, excepto uno, en el cual se colocó un halo craneano inicialmente. En los cinco casos tratados de modo conservador, se observaron buenos resultados, con estabilidad del segmento en las radiografías funcionales, ausencia de limitación de movimiento y ausencia de dolor. El paciente que fue tratado con halo presentaba tetraplejía parcial FRANKEL C y fracturas asociadas (C5, C6, L1 y L2) y no presentó mejora del cuadro neurológico, verificándose limitación del movimiento cenvical. Destacamos además la importancia de la investigación activa de lesiones en la transición occipitocenvical, principalmente en los casos provenientes de trauma de alta energía, pues además de las implicaciones clínicas de la detección precoz de la fractura, el mejor empleo de los exámenes subsidiarios ha sido un factor relevante para el aumento de la incidencia de fracturas del cóndilo occipital. Como es históricamente relatado, el tratamiento conservador aún es eficaz y con bajo índice de complicaciones, y el tratamiento quirúrgico debe ser indicado en casos con inestabilidad definida.

Descriptores: Hueso occipital; Traumatismos cráneo-cerebrales; Humanos.

INTRODUÇÃO

Fratura de côndilo occipital foi inicialmente citada na literatura em $1817^{1}$ e somente em 1900 encontra-se o segundo caso em publicação médica. ${ }^{2}$ Tempos depois, em 1974, mais seis casos de fratura de côndilo occipital foram apresentados e em 1978, mais dois casos. Desde então, poucos casos têm sido descritos na literatura.

Estudos sobre essas fraturas foram iniciados por Ahgren e
Dahierup $^{3}$ em 1962 e 1964 e Schliak e Schaefer. ${ }^{4}$ Em 1957, Werne ${ }^{5}$ examinou espécimes de 28 indivíduos com fraturas de base de crânio e fraturas dos côndilos occipitais, por compressão ou avulsão, gerando um estudo sobre anatomia, aspectos radiológicos e análise biomecânica dos ligamentos da porção superior da coluna cervical. Em 1986, Dvorak e Panjabi 6 publicaram seu estudo de anatomia funcional dos ligamentos alares e, em 1988, Anderson e Montesano ${ }^{7}$ publicaram uma classificação

1. Instituto de Ortopedia eTraumatologia do Hospital das Clínicas da Faculdade de Medicina da Universidade de São Paulo, São Paulo, SP, Brasil.

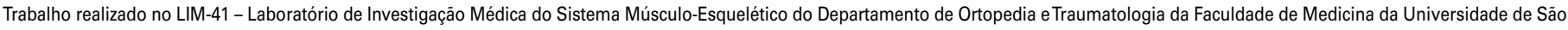
Paulo. São Paulo, SP. Brasil.

Correspondência: Rua Dr. Ovídio Pires de Campos, 333. São Paulo, SP, Brasil. 05416-010. aacristante@uol.com.br 
que correlaciona mecanismo de trauma com morfologia de fratura, além de identificar possível instabilidade ligamentar.

Os côndilos occipitais são constituídos de quatro partes: basilares, laterais ou condilares e escamosa. ${ }^{8}$ Articulando-se com os côndilos occipitais está a primeira vértebra cervical, o atlas, e este, com o áxis. Nesta articulação se dá aproximadamente $50 \%$ do movimento de rotação da cabeça. Ao seguimento cervical superior $(\mathrm{C} 1 \mathrm{e}$ C2) e occipício chamamos unidade cervicocraniana. ${ }^{9}$ Os movimentos possíveis no segmento occiptoatlantoaxial são estabilizados por um conjunto de ligamentos e pela membrana tectorial. A membrana tectorial, o ligamento nucal, o ligamento longitudinal posterior e o ligamento cruciforme conferem estabilidade regional ao movimento de flexão da porção superior da coluna cervical. A estabilidade dos movimentos de rotação e inclinação lateral é conferida pelos ligamentos alares. O Atlas move-se na mesma direção da inclinação, porém não se observa sua rotação. O movimento de extensão da parte superior da coluna cervical é limitado principalmente pela porção transversa dos ligamentos alares. ${ }^{6}$

As fraturas do côndilo occipital classicamente são de tratamento conservador, com baixas taxas de complicação (dor, limitação de movimento), evoluindo de forma bastante favorável. A despeito deste fato, são de difícil diagnóstico se não houver a suspeita da existência das mesmas e se não for utilizado método diagnóstico adequado para sua identificação. A busca ativa por tais lesões deve ser incisiva. ${ }^{10-13}$ O objetivo do presente trabalho é apresentar seis casos de fratura de côndilo occipital atendidos em nosso serviço, dando sequência ao levantamento epidemiológico iniciado realizado em 2000. Além disso, reforçar a suspeita diagnóstica em casos de trauma crânio-encefálicos e em vítimas de colisão de alta energia e alertar para a necessidade de estudo tomográfico da transição occipito cervical na suspeita.

\section{METODOLOGIA}

De 2001 a 2011 foram diagnosticados em nosso serviço seis casos de fratura de côndilo occipital. Foi feita uma análise retrospectiva dos prontuários quanto a(o):

- Sexo;

- Idade;

- Mecanismo de trauma;

- Classificação das fraturas segundo a classificação de Anderson e Montesano;

- Tratamento a que foram submetidos;

- Resultado do tratamento avaliando-se estabilidade do segmento nas radiografias funcionais, limitação de movimento e dor pós-tratamento.

O tempo médio de segmento foi de 2 anos.

\section{Classificação de Anderson e Montesano:}

Tipo I: Fratura impactada do côndilo occipital; mecanismo de trauma: carga axial do crânio sobre o Atlas. Há cominução do côndilo occipital sem ou com mínimo desvio dos fragmentos em direção ao forame magno. Os ligamentos encontram-se íntegros, o que garante a estabilidade.

Tipo II: Fratura do côndilo occipital que estende para a base do crânio com traço em direção ao forame magno. Mecanismo: trauma direto. Ligamentos íntegros, fratura estável.

Tipo III: Fratura avulsão do côndilo occipital pelo ligamento alar. Mecanismo: rotação ou inclinação lateral da cabeça ou associação dos dois movimentos. Neste caso, devido à lesão do ligamento alar e da membrana tectorial, observa-se uma lesão potencialmente instável. (Figuras 1-3)

\section{RESULTADOS}

A análise retrospectiva dos prontuários dos seis pacientes atendidos em nosso serviço com diagnóstico de fratura de côndilo occipital no período de 2001 a 2011 revelou três casos de fratura tipo I, dois casos do tipo II e um caso do tipo III, segundo a classificação de Anderson e Montesano. (Tabela 1)

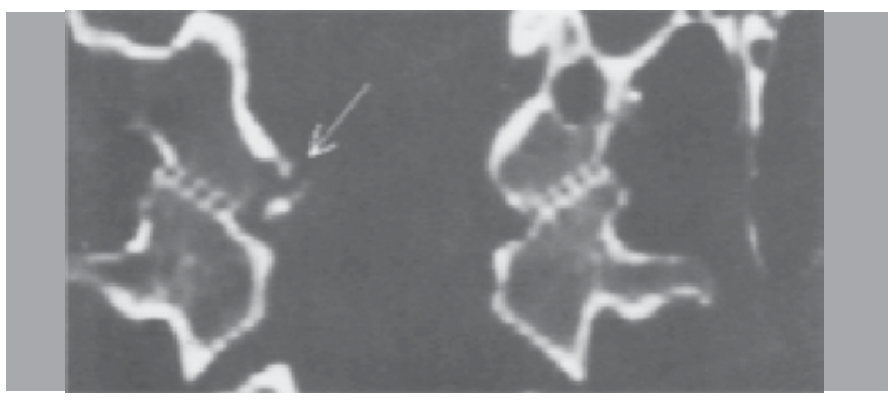

Figura 1. Fratura tipo $\mathrm{I}$.

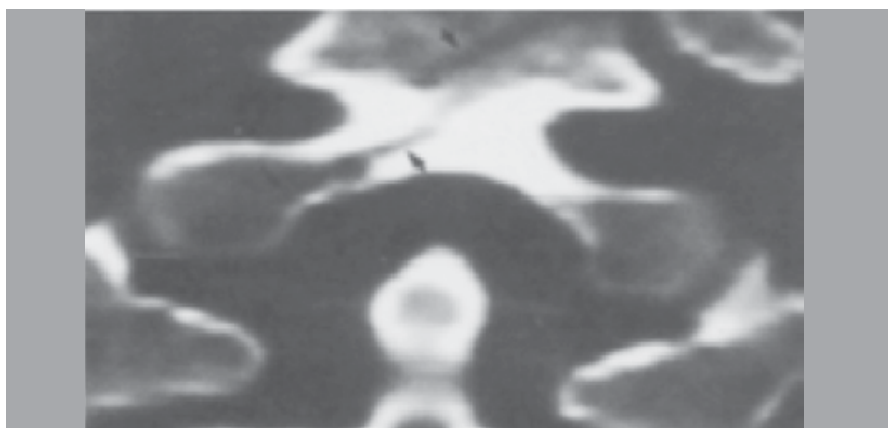

Figura 2. Fratura tipo II.

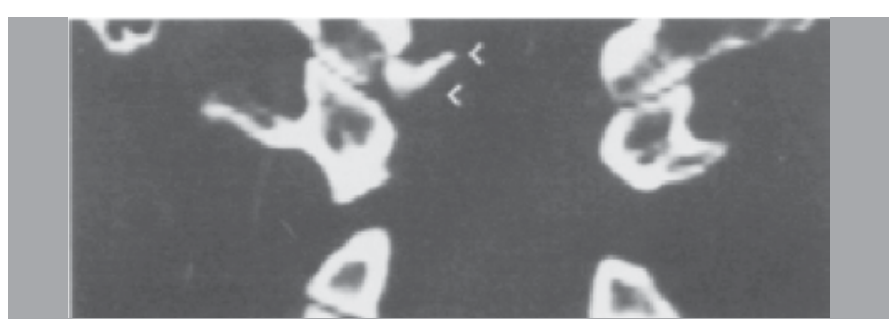

Figura 3. Fratura tipo III.

Tabela 1. Descriçao dos casos com diagnóstico de fratura de côndilo occiptal.

\begin{tabular}{c|c|c|c|c|c|c}
\hline Paciente & 1 & 2 & 3 & 4 & 5 & 6 \\
\hline Sexo & $\mathrm{M}$ & $\mathrm{M}$ & $\mathrm{F}$ & $\mathrm{M}$ & $\mathrm{M}$ & $\mathrm{M}$ \\
\hline Idade & 24 & 33 & 17 & 22 & 42 & 54 \\
\hline Mecanismo & $\mathrm{AA}$ & $\mathrm{AM}$ & $\mathrm{QA}$ & $\mathrm{AA}$ & $\mathrm{A}$ & $\mathrm{QA}$ \\
\hline Tipo & $\mathrm{I}$ & $\mathrm{I}$ & $\mathrm{II}$ & $\mathrm{I}$ & $\mathrm{I}$ & $\mathrm{I}$ \\
\hline $\begin{array}{c}\text { Fraturas } \\
\text { associadas }\end{array}$ & - & - & - & $\mathrm{C} 5, \mathrm{C} 6, \mathrm{~L} 1 \mathrm{e} \mathrm{L2}$ & - & - \\
\hline Déficits & - & - & - & Frankel C & - & $\mathrm{LPB}$ \\
\hline Resultado & $\mathrm{N}$ & $\mathrm{N}$ & $\mathrm{N}$ & $\mathrm{D} / \mathrm{D}$ & $\mathrm{N}$ & $\mathrm{N}$
\end{tabular}

$\mathrm{M}=$ masculino; $\mathrm{F}=$ feminino; $\mathrm{AA}=$ acidente automobilístico; $\mathrm{AM}=$ acidente motociclístico; $\mathrm{QA}=$ queda de altura; $A=$ atropelamento; $L P B=$ lesão de plexo braquial; $N=$ normal; $D / D=$ déficit e dor.

A paciente 3, mesmo apresentando fratura tipo III, foi escolhido o tratamento conservador. Durante o seguimento houve consolidação óssea em quatro meses de seguimento, com boa evolução clínica.

O paciente 4 apresentava lesões associadas (fraturas de C5, C6, L1 e L2). Ao exame neurológico, apresentava força motora grau 2 bilateral de $\mathrm{C} 7$ a S1, e sensitivo parcial a partir do mesmo nível. Inicialmente, havia programação de tratamento cirúrgico para as fraturas de $\mathrm{C} 5$ e $\mathrm{C} 6$ e fora realizado halo craniano para alinhamento vertebral. Entretanto, este fora substituído em uma semana por colar cervical visto que o paciente apresentou distúrbio ventilatório e necessitou de cuidados semi-intensivos, terminando o tratamento com órtese. Déficit mantido no seguimento. 
O paciente 6 apresentava lesão de plexo braquial de C5 a C7 (FMG 0) à esquerda, confirmada por eletroneuromiografia; déficit resolvido totalmente em seis meses de seguimento.

Os demais pacientes apresentavam fratura unilateral de côndilo occipital sem outros achados e sem alterações neurológicas ao exame físico.

Todos os casos tratados conservadoramente evoluíram sem dor, com estabilidade do segmento nas radiografias funcionais e sem limitação de movimento. O paciente submetido ao halo evoluiu com dor leve e limitação de movimento cervical, além da manutenção do déficit neurológico.

\section{DISCUSSÃO}

Apesar da pequena casuística demonstrada, evidenciamos que as fraturas de côndilo occipital são mais comuns em pacientes masculinos, entre 20 e 30 anos, em geral decorrente de traumas de alta energia como acidentes de trânsito, quedas de altura, o que corresponde aos estudos epidemiológicos prévios. . $^{911,13,14} \mathrm{O}$ trauma crânio-encefálico acompanha a grande maioria dessas fraturas, 0 que também dificulta seu diagnóstico e sendo muitas vezes o responsável pelo óbito dos mesmos. Cabe ressaltar ainda a possível associação dessas lesões às fraturas de vértebras cervicais.

O quadro clínico das fraturas do côndilo occipital pode variar desde uma dor na face posterior do pescoço, de espasmo da musculatura paravertebral cervical simulando um torcicolo, como pode se observar um quadro de dor cervical com paralisia dos IX, X e XI pares cranianos, em raros casos. Esta sintomatologia é atribuída ao grande potencial apresentado por esse tipo de fratura para o comprometimento do canal hipoglosso que se encontra na base do côndilo occipital. Devido à necessidade de exames específicos, não rara- mente estas fraturas podem passar despercebidas ao exame inicial. ${ }^{13}$

A visualização deste tipo de fratura com as técnicas radiográficas habituais é extremamente difícil, sendo comumente necessário o uso de tomografia, que é a técnica de escolha para seu diagnóstico. Cortes finos (1,2mmx1mm) permitem ótimo estudo de morfologia óssea e ligamentar, além de permitir excelente reconstrução tridimensional. ${ }^{15}$

O tratamento conservador das fraturas de côndilo occipital evolui com bons resultados, ficando o paciente livre da dor cervical e mantendo o arco total de movimento do segmento envolvido após três meses de tratamento. Preconizam-se o uso de colar tipo Philadelphia para casos classificados como do tipo I e II de Anderson e Montesano, ${ }^{7}$ e uma imobilização mais rígida, como halo gesso ou gesso Minerva pra as fraturas classificadas como tipo III, cabendo ainda o tratamento cirúrgico em casos de instabilidade persistente.

\section{CONSIDERAÇÕES FINAIS}

A despeito do quadro clínico inicial, as fraturas de côndilo occipital devem ser investigadas na presença de trauma crânio-encefálico de alta energia. Os exames radiológicos convencionais devem ser acrescidos da tomografia na suspeita clínica. Fraturas associadas devem ser descartadas e evidentemente tratadas. Déficit neurológico é raro e geralmente constituídos de neuropraxia. O tratamento conservador na maioria das vezes é bem indicado e apresenta bons resultados, podendo sempre ser introduzido, exceto quando há instabilidade refratária.

Todos os autores declaram não haver nenhum potencial conflito de interesses referente a este artigo.

\section{REFERÊNCIAS}

1. Bell C. Surgical Observations. Middlesex Hosp J. 1817;4:469-70

2. Kissinger P. Luxationfraktur in atlanto-occipital Gelenke. Zentralbl Chirurgie 1900;37:933-4.

3. Ahlgren P, Dahlerup JV. Fracture of the occipital condyle. A new case of isolated fracture. Fortschr Geb Rontgenstr Nuklearmed. 1964;101:202-4.

4. Schliack H, Schaefer P. Hypoglossal and accessory nerve paralysis in a fracture of the occipital condyle. Nervenarzt. 1965:36(8):362-4.

5. Werne S. Studies in spontaneous atlas dislocation. Acta Orthop Scand Suppl. 1957;23:1-150.

6. Dvorak J, Panjabi MM. Functional anatomy of the alar ligaments. Spine (Phila Pa 1976). 1987:12(2):183-9.

7. Anderson PA, Montesano PX. Morphology and treatment of occipital condyle fractures. Spine (Phila Pa 1976). 1988;13(7):731-6.

8. Lockhart RD, Hamilton GF. Anatomy of the human boby. London: Faber and Faber; 1959

9. Spencer JA, Yeakley JW, Kaufman HH. Fracture of the occipital condyle. Neurosurgery. 1984;15(1):101-3
10. Goldstein SJ, Woodring JH, Young AB. Occipital condyle fracture associated with cervical spine injury. Surg Neurol. 1982;17(5):350-2.

11. Harding-Smith J, Maclntosh PK, Sherbon KJ. Fracture of the occipital condyle. A case report and review of the literature. J Bone Joint Surg Am. 1981;63(7):1170-1.

12. Peeters $F$, Verbeeten $B$. Evaluation of occipital condyle fracture and atlantic fracture, two uncommon complications of cranio-vertebral trauma. Rofo. 1983;138(5):631-3.

13. Bridgman SA, McNab W. Traumatic occipital condyle fracture, multiple cranial nerve palsies, and torticollis: a case report and review of the literature. Surg Neurol. 1992;38(2):152-6.

14. Bolender N, Cromwell LD, Wendling L. Fracture of the occipital condyle. AJR Am J Roentgenol. 1978;131(4):729-31.

15. Bloom Al, Neeman Z, Slasky BS, Floman Y, Milgrom M, Rivkind A, et al. Fracture of the occipital condyles and associated craniocervical ligament injury: incidence, CT imaging and implications. Clin Radiol. 1997;52(3):198-202. 